\title{
Téoros
}

Revue de recherche en tourisme

\section{L'hébergement et la clientèle de congrès}

\section{Gustav Bamatter}

Volume 8, numéro 2, juillet 1989

Hébergement et tourisme

URI : https://id.erudit.org/iderudit/1080320ar

DOI : https://doi.org/10.7202/1080320ar

Aller au sommaire du numéro

Éditeur(s)

Université du Québec à Montréal

ISSN

0712-8657 (imprimé)

1923-2705 (numérique)

Découvrir la revue

Citer cet article

Bamatter, G. (1989). L'hébergement et la clientèle de congrès. Téoros, 8(2),

13-15. https://doi.org/10.7202/1080320ar d'utilisation que vous pouvez consulter en ligne.

https://apropos.erudit.org/fr/usagers/politique-dutilisation/ 


\section{L'hébergement et la clien- tèle de congrès}

Les congrès, d'une façon générale, relèvent d'une de deux catégories, soit:

Un congrès où les personnes tiennent leurs réunions et hébergent dans un seul hổtel;

Un congres pour lequel participent plusieurs hôtels alors que les réunions ont licu dans un cndroit à l'extérieur, tel que le Stade, le Palais des Congrès, une salle d'eglise, un aréna, etc..

\section{Congrès uni-hôtel}

Nous allons d'abord traiter du congrès uni-hôtel et réunions. Le succès de cescongrès, majoritairement organisés et réservés des mois ou des années à l'avance, dépend presque toujours des décisions prises par l'hôtel au moment de la signature des ententes avec les organisateurs. Les deux parties, organisateurs et hôtel, doivent. afin de s'assurer d'un déroulement sans probleme, couvrir un nombre de points spécifiques. De plus, il est nécessaire que le directeur de l'hébergement participe à ces discussions, puisque les résultats affecteront directement son service.

a) Les aspects essentiels à considérer.

Parmi les aspects essentiels à considérer, nous avons retenu les points suivants:

- Où s'est tenu le dernier congrès de cet organisme et quel était le pourcentage de fiabilité à occuper les chambres réservées nombre de nuitées vendues versus le nombre de nuitées réservées)? Normalement, le directeur(trice) commercial(e) du dernier endroit où l'organisme a tenu sa réunion vous donnera cette information, surtout si elle est négative. On vous dira ćgalement s'il y a eu des problèmes majeurs, tels que dommages aux chambres, bruit excessif, comportement inacceptable vis-à-vis les autres clients, difficulté de paiement, etc..

- Il faut obtenir, de la part des congressistes, une évaluation réaliste du nombre de chambres prévues pour l'événement. Il vous appartient de décider si vous voulez fournir toutes vos chambres ou une partic seulement.

- Dequelle maniere se feront les réservations? Individuellement avec une carte de réservation et garantie par une carte de crédil ou envoi monétaire; il faut obtenir la liste d'hé- bergement de l'organisation avec une garantie minimale de réservation de la premiere nuit. Dans la planification de l'événement, il faut aussi tenir compte de la clientèle habituclle dont la demande de disponibilité de chambres peut être difficilement refuséc. II est bon alors de vérifier les possibilités existantes chez les hôtels les plus rapprochés au cas oủ vous manqueriez de chambres au demier moment.

- L'importance d'ćtablir un échéancier réaliste pour les réservations ne peut être surestimée. Prenons un exemple. Un congrès a lieu durant le mois de juillet à Montréal, soit durant la semaine du Grand Prix Automobile. Vous savez que toutes les chambres de la réservation originale qui ne seront pas occupées à une date limite fixée à 20 jours de louverture ducongrès seront vendues comme des petits pains chauds dès qu'elles devien= dront disponibles. Ainsi, une date limite de 20 jours sera amplement sécuritaire pour votre entreprise pour afficher complet. Par ailleurs, si vous obtenez un congrès similaire dans la ville de Québec à la mi-novembre, soit en trè basse saison, vous pourrez être à peu près certain que vous ne pourrez vendre ces chambres sauf avee un pré-avis de 90 jours; ce que les organisateurs trouveront excessivement exigeant. Dans un tel cas, il faut ré-examiner, avec le responsable du congrès, la situation des réservations à 90 jours de l'événement. Les prêvisions originales peuvent demeurer inchangées ou encore être révisćes à la baisse. Oocasionnellement, le nombre de chambres retenues est réduit unilatéralement par le responsable de l'hôtel, si ce dernier a besoin de ces chambres pour d'autres clients et est persuadé que le congrès n'honorera pas sa réservation au complet. La plupart des directcurs d'hôtels ont déjà vécu le cauchemar d'un "overbooking" ou de la surréservation! II est malheureusement presqu'impossible de vendre toutes les chambres de l'hôtel sans accepter ce risque calculé.

- Les organisateurs devront aviser l'hôtel dès que possible de leurs besoins specifiques d'hébergement. Ainsi, par exemple, y a-t-il des V.I.P. (personnes très importantes) parmi les congressistes? Combien de suites d'hospitalité seront requises? Quelles sont les demandes spéciales (gens qui ont peur des étages supérieurs, facilités sportives 


\section{ouvertes plus longtemps...?}

- Enfin, un dernier point. Les liens professionnels que la personne en charge du crédit de I'hôtel aura su établir avec la personne en charge des finances de l'organisation amoindriront sans aucun doute les difficultés de facturation.

b) Autres aspects de l'organisation à considérer el à resoudre.

Il est difficile pour quelqu'un qui oeuvre hors du domaine de l'hôtellerie de s'imaginer l'extrême confusion qui peut resulter d'un enregistrement mal planifié pour un congrès majeur $(300 \mathrm{cham-}$ bres et plus).

Les services de la réception et des réservations doivent d'abord s'assurer que tous les clients qui détiennent des réservations de 18 hoo et/ou garanties, soient pré-enregistrées et que tous les. détails soient inserits à l'ordinateur, ou manuellement. Il faut déterminer à quel moment se situeront les heures de pointe pour lenregistrement. S'il s'agit d'un congrès international, il faut contacter les lignes aćriennes et confirmer les heures d'arrivée. Les mêmes démarches sappliquent pour les gens qui voyagent par train. Selon notre expérience personnelle, il ne fait aucun doute qu'un groupe qui remplit un Boeing 747 aux trois-quart et qui arrive à votre hôtel mal préparé vous rendra absolument furicux.

Selon que le congres national ou provincial, it faut done tenir compte des moyens de transport des congressistes, c'est-à-dire via le train ou l'avion, ou encore en automobile; il faut aussi noter le jour de la semaine où le plus grand nombre de congressistes debarqueront. Par excmple, il est presque assuré que les congressistes qui doivent arriver le vendredi pour un congrès provincial de fin de semaine seront en retard. Les congressistes auront quitté leur travail entre $15 \mathrm{~h} 00$ et $17 \mathrm{~h} 00$ et arriveront tous après $19 h 00$, fatigués de leur joumée et plutôt impatients de retrouver leur chambre et prendre un verre avec les amis. Par contre, si l'enregistrement est le dimanche, les mêmes personnes arriveront à compter de midi, ne se sentiront pas pressées et seront beaucoup plus relaxes.

Si l'espace de comptoir de la réception est restreint par rapport au nombre de congressistes, lat possibilité d'ouvrir des comptoirs satellites dans le hall d'entrée doit être retenue. Ces comptoirs seront clairement indiquess par lettres alphabetiques et opérés par deux personnes ayant tous les dossiers en mains. Comme ça, M. et Mme Laliberté iront s' inscrire au comptoir indiqué AM. Leur nom s'y trouvera dejă, avec tous les détails; une chambre leur sera assignee selon leur preférence et une impression de leur carte de crédit sera faite. L'entré̉e de cette donnéc sur ordinateur, si cest une opeŕration informatisćc, peut se faire tout de suite si des appareils satel- lites d'entrée de données sont disponibles, ou encore il est possible de simplement inscrire l'information sur une fiche et d'apporter ces fiches régulièrement à la réception pour l'entréc de données. Il faudrágalement prévvoir unespace près de la porte d'entrée pour l'enregistrement des congressistes par les organisateurs du congress; on doitaussi maximiser beaucoup l'échange dinformation entre les responsables. Lorsque la clé est présentée au elient, il est habituellement utile de lui remettre une lettre lui souhaitant la bienvenue et lui donnant quelques informations pratiques qui elimineront considérablement les appels au service d'information de l'hötel.

D'autre part, un bon nombre de chasseurs devra etre prévu afin d'assurer le dégagement continuel vers les chambres. Si l'on considere que chaque congressiste a 2-3 valises, il est facile d'imaginer l'etat du hall d'entrée quand 200-300 personnes viennent d'arriver. Cela dit, il n'est pas inutile qu'un des cadres superieurs de l'hótel se tienne en permanence dans le hall d'entrée, durant les heures de trafic majeur, afin de résoudre tout problème urgent. Il faut aussi s'assurer que les nouveaux arrivés déplacent immédiatement leur véhicule apres avoir déchargé les valises; si cela nest pas fait, vous risquez fort d'irriter les clients qui suivent.

Les machines distributrices de glace et sodas devront toutes être vérifiecs et remplies avant l'arrivée du congrès. Surtout la glace ... et si par malheur il en manque, le client ne manquera de vous faire remarquer que lorsquil tetait de passage au Motel du Sapin Ver - établissement beaucoup plus petit et moins dispendieux que le vôtre - il y en avait de la glace en quantité et qu'il est incompréhensible qu'avec vos 4,5 ou 6 fleurs de lys...

Les ascenseurs dans les hôtels à plusieursétages demeurent toujours le tallon d'Achille d'un établissement. II faut quils soient en assez grand nombre, fiables et résistant à un usage peu habituel et même oxcasionnellement abusif des passagers. Un nouveau terme américain suggèreque l'ascenseut soit "idiot proof" (à l'épreuve des idiots)!

Vos Club de santé et Centre Sportif devront ćtre prêts à recevoir un surplus de réservations, toute comprimées entre les heures de conférence et le dîner, ou très tôt le matin.

Il faut donner une attention toute spéciale à la programmation des repas. Si votre hôtel est remplie à pleine capacité de congressistes, et que la majorité des repas sont servis à l'occasion de banquets, il se peut que vous ayez des problèmes de logistique au moment des petits-déjeuners, cest-a-dire une ligne d'attente très longue et conséquemment, en une clientèle mal servie et malheurcuse, A vous d'ouvrir des facilités de repas additionnelles.
Il est maintenant une heure du matin et la plupart des congressistes sont enregistrés. Il en reste seulement une vingtaine â arriver et vous çtes déjà convaincu qu'ils ne se présenteront pas tous. Des clients de passage sont au comptoir et veulent avoir une chambre, mais n'ont pas de réservation. La tentation de vendre une des chambres réservec au congrès est forte. Le superviseur de nuit veut démontrer à son patron qu'il peut maximiser les revenus et augmenter le tarif moyen. Le jeu du "overbooking" (sur réservation) commence. La pluparı des grands hớtelss'y adonnent, mais habituellement de façon très conservatrice. Parexemple, si une personne arrive avec une réservation garantic et qu'il ne reste plus de chambres, la façon la plus courante de régler le problème est de l'envoyer, aux frais dé lhôtel (transport et hébergement), à un établissement voisin jouissant dune classification egale ou supéricure. Malheureusement, il arrive souvent que ce client soit un de vos clients réguliers quí non seulement prendra mal oc changement dans ses habitudes, mais décidera de ne plus fréquenter votre établissement. Malgré le fait qu'on se perde en excuses et qu'on fasse tout ce qui est humainement possible pour parer à ces inconvénients, il faut admetire que celte pratique en cat une à double tranchant. L'equation "revenu additionnel maintenant versus revenu perdu a long terme" savère souvent négatif.

Le travail du verrificateur de nuit qui doit reconcilier toutes les transactions des chambres et des restaurants n'est évidemment pas une lâche facile quand l'hobtel affiche complet. Cependant, une bonne qualification, de mểme que des fiches tenues à jour la rendront beaucoup plus aisée.

Le congrès est maintenant terminé et il est $12 \mathrm{~h} 45$. Lheure du départ de votre hôtel, affiché dans toutes les chambres, est 13h00; $90 \%$ des congressistes seront devant la caisse entre $12 \mathrm{~h} 50 \mathrm{et}$ $13 \mathrm{~h} 00$, heure pendant laquelle les caissiers(ères) feront une depression nerveuse devant les lignes d'attente de personnes impatientes a partir. Encore une fois, une bonne planification peut éviter le plus gros du probleme. Quelques solutions soni à prévoir: ainsi, des départs "express" dans le cas où le client a déjà signé lä carte de crédit et reçoit la justification écrite par la poste; présentation de la facture durant la dernière nuit en la glissant sous la porte de la chambre pour vérification: possibilité de voir la facture sur l'écran du téléviseur dans la chambre dụ client et même de l'approuver électroniquement: un autre moyen, employé encore fréquemment dans les hôtels européens est de glisser dans la chambre du clicnt à midi le jour précédent le départ une petite "invitation" à visiter lạ caisse durant la soirée afin de régler le compte et ainsi êviter la congestion du lendemain. 
Évidemment, il est essentiel d'avoir un bon contrôle des dépenses du lendemain matin pour s'assurer que le client paye la facture du petitdéjeunerqui ne peut plus être chargé à sa chambre.

\section{2- Congrès multi-hôtels avec réunions ailleurs, le cas du Palais des Congrès}

On rencontre cette situation nomalement seulement pour des congrès de trè̀s grande envergure. Sans une planification méticuleuse et une parfaite coopération entre plusieurs hôtels et le Palais des Congrès, il est presque impossible d'obtenir du succès pour ce genre de congrès. Prenons comme exemple l'Association internationale des clairvoyants du monde qui tient un congrès tous les 3 ans dans l'un des quatre coins du monde. Ils ont besoin pour leur congrès d'un nombre approximatif de 3500 chambres. Un des délégués du Palais des Congrès s'envole à Lhasa au Tibet pour rencontrer la personne ressource de cette organisation et revient en demandant aux hôteliers de Montréal quelle sera la disponibilite de leurs chambres entre le 6 et le 12 septembre 1994. Il est déjă au courant que le Palais des Congrès est disponible. Pour des raisons d'efficacité de transportet dans le but de faciliter le congrès, on decide de partager les 3500 chambres entre 10 hôtels du centre-ville. Chacun de ces hôtels accepte de réserver tentativement un certain nombre de chambres. Notre délégué du Palais des Congrès, proposition en mains, retourne à Lhasa avec une gamme complète d'informations pertinentes au congrès et présente son cas. Pour simplifier la situation, nous allons prendre pour acquis que la proposition sort vainqueur parmi les 12 soumissions et que le congrès sera tenu à Montréal du 6 au 12 septembre 1994.

Notre heureux délégué revient à Montréal, en réalisant que la partic facile de la tâche est complétée et que la partie difficile va commencer. Le Comité qui sera mis sur pied devra continuellement communiquer avec les organisateurs, afin de s'assurer que tous les points soient mis sur les " $i$ ". Ainsi, le transport entre les hôtels et le Palais devra être mis sur pied. Des informations pertinentes devront être remises à chaque client. Les congressistes devront être répartis parmi les 10 hôtels, soit par liste de préférence ou selon le choix des organisateurs. Chaque congressiste recevra une confirmation écrite de "son" hôtel. On devra planifier des arrangements pour enregistrer tous ces congressistes au Palais ou à chaque hôtel participant. Si nécessaire, il devrait y avoir des interprètes sur place afin d'éviter des difficultes de communication. Les particularités sociales, culturelles, religieuses ou gastronomiques de certains congressistes devront être portées à l'attention de tout le personnel des hôtels. La tenue mểme du congrès, une fois commencée, se déroule de la même façon, cn ce qui concerne l'hébergement des congressistes, que le congrès uni-hótel et avec les mẻmes besoins de planification méticuleux et d"attention de détail.

Finalement, soulignons quelques points essentiels des qualités de l'hébergement. Les chambres devront évidemment être propres et confortables; les directions pour se rendre aux chambres devront être claires. Sur chaque étage ou section, des lits pliants et des lits d'enfants devront être disponibles, ainsi que des planches et fers à repasser s'il n'y en a pas déjà dans les chambres. A part l'information générale des services de l'hôtel, il devrail y avoir de l'information sur ce qu"il faut faire en cas d'incendie, d'urgence médicale, de besoin spécial concernant les enfants; de même que de l'information sur les services religieux, le service des objets perdus et celui de la sécurité de l'hôtel. Il faut surtout que le congressiste se sente à l'aise et confortable afin qu'il conserve sa bonne humeur et sa joie d'čtre votre visiteur. 